\title{
A Survey on Feature Extraction Techniques
}

\author{
Ayushi \\ Manav Bharati University,Solan
}

\begin{abstract}
Face recognition is very important in computer vision. For human being it is easy to identify human face in any posture but it is not an easy task for systems. Feature extraction is useful technique for recognizing faces through systems. It is used for security, criminal records or identification, verification of person, etc. In face images there are variety of face posture. The question is, How to recognize the face? In this paper, discuss certain approaches for face recognition by using feature extraction along with the issues and the recommendations as to which approach is better and suitable.
\end{abstract}

\section{Keywords}

Features Extraction, Face Recognition, Issues, Recommendations.

\section{INTRODUCTION}

In the recent years Automatic Face Recognition have received much attention, due to which it is becoming a challenging task. Face Recognition has its applications in different fields such as video indexing, feature extraction, law enforcement, and other security applications. There is no technique that provides a robust solution to all situations and different applications that face recognition may encounter [1].

Face Recognition has 2 senses:

1. Biometric identification by scanning a person's face and matching it against a library of known faces; "they used face recognition to spot known terrorists"

2. The visual perception of familiar faces.

It is a type of biometric software application that enables identification of a person from an image of his or her face by comparing selected facial features from the image and a facial database. These systems work with numeric codes called faceprints. Such systems identify 80 nodal points on a human face, in which nodal points are end points used to measure variables of a person's face, such as the length or width of the nose, the depth of the eye sockets and the shape of the cheekbones. These systems work by capturing data for nodal points on a digital image of an individual's face and storing the resulting data as a faceprint. The faceprint can then be used as a basis for comparison with data captured from faces in an image or video.

It is typically used in security systems and can be compared to other biometrics such as fingerprint or eye iris recognition systems. Recognition algorithms can be divided into two main approaches:

Geometric: This looks at distinguishing features.

Photometric: This is a statistical approach that distills an image into values and compares the values with templates to eliminate variances.

\section{ISSUES IN FACE RECOGNATION}

A. Face Identification: Face Identification is a main issue in face recognition. It is used in credit card verification, criminal records, and many security systems. Face identification is used to identify the face from the whole image. Sometimes, images are affected by noise, shadow, surrounding factor like light. That time correct identification of face is very difficult. So in each and every case face identification must be correct.

B. Feature Localization: In face recognition another issue is feature localization. The face of a person in different images is different because of his distinct face expression (like: smile, angry, tensed, neutral, shock etc), variety of posture, and few other factors like: hairs, goggles, hat etc. In face recognition some features like: eyes, nose, and mouth are considered. In order to recognize face features must have proper visualization.

C. Corner Point Extraction: To recognize the face by extracting features i.e. eyes, nose, mouth. In different location of features in distinct images, the corner points of the features are extracted because corner points are same in all images of a person. So extraction of the corner points of features is very important, it should be correct.

D. Scaling: In scaling, the distance between center points of eyes, corner points of eye, center of eye and nose, center of nose and mouth, and interior corner points of eyes are measure. So, one must keep in mind that the scaling should be accurate.

\section{STUDY OF DIFFERENTAPPROCHES}

Many researchers have given distinct approaches for feature extraction in face recognition. The main issues considered by maximum researchers are localization of face, variety of posture, surrounding factors, feature extraction etc. In this paper discuss these approaches.

\subsection{Subspaces Approach}

Teja et.al [2] had proposed subspaces techniques for face recognition. He investigated the pre-processing of images as a primary step to reduce the error rates. In this paper, implemented the updated methods of Principle Component Analysis, Linear Discriminant Analysis using subspaces techniques. It also used a standard test set of images by which author computed the false Acceptances Rates (FAR) and false Rejection Rates (FRR). For this performance of image processing techniques dependent on the pre-processing steps. By using the proposed methods the Equal Error Rates (EER) of Eigenface and fisherface methods can be reduced. The testing on distinct size, resolution and number of pixels of images using three subspace techniques i,e eigenfaces, fisrherfaces and probabilistic eigenfaces approach proposed in [3]. In this paper, the relationship between accuracy of recognition and image resolution, which enhance the performance in comparison individual methods. A face 
recognition technique that is based on Appearance Based subspaces projection introduced by [4]. For this all the subspace projection algorithms i.e. PCA, LDA, ICA and NMF are explored which are used to reduce the dimensionality of high dimension face image data. The results presented and analyzed after simulation of these techniques are implemented with ORL face dataset. Also the four techniques are compared in recognition performance for FR.

\subsection{Principal Component Analysis (PCA)}

Principal Component Analysis is used to extract the features to recognize the faces. Given an s-dimensional vector representation of each face in a training set of images, Principal Component Analysis (PCA) tends to find a tdimensional subspace whose basis vectors correspond to the maximum variance direction in the original image space. Patil, A.M. et. Al [5] proposed an appearance based eigenface technique in which the related information of human faces have been extracted and then encoded by it. The encoded information compared with Indian Face Database and encodes the variation between encoded Face images and stored database by face space. In this technique face space is used to project the face images which are computed by eigenvectors. A method has been proposed, in which "No Human Touch" technology is used [6]. This method involves three steps i.e.: i) Preprocessing, ii) feature extraction using curvelet, PCA with Singular value decomposition iii) classification and recognition using Bayes' algorithm.
For enhancing the face recognition rate from few samples of available images the combination of PCA with Singular Value Decomposition and Bayes Classifier is used. This method provides a fast computation in constrained environment. In paper [7] introduced a human face recognition algorithm based on greedy kernel principal component analysis (GKPCA). It introduces the need of online quick face recognition, in which fast wavelet transform is used to decompose the complicated human face images then the training set is reduced by the Greedy algorithm. Kernel Principal Component Analysis (KPCA) extracted the feature of low frequency images i.e. recognized by the support vector machine(SVM).This algorithm provide the reducing training time with high recognition rate. The ORL database of Faces consists of monochrome images different times, varying the lighting, facial expressions (open / closed eyes, smiling / not smiling and facial details (glasses / no glasses), All the images were taken against a dark homogeneous background. The Kernel-PCA with Gaussian function can provide a good recognition rate i.e.similar to PCA and higher than KernelPCA with polynomial function [8].

RECOMMENDATIONS: Rajkumar. N et.al introduced a method for fast computation in constrained environment. The drawback of PCA is when the input space increases than it fails and in case of twins it suffers from the problem of discrimination of faces. Whereas kernel PCA extracted the features from the low frequency images.

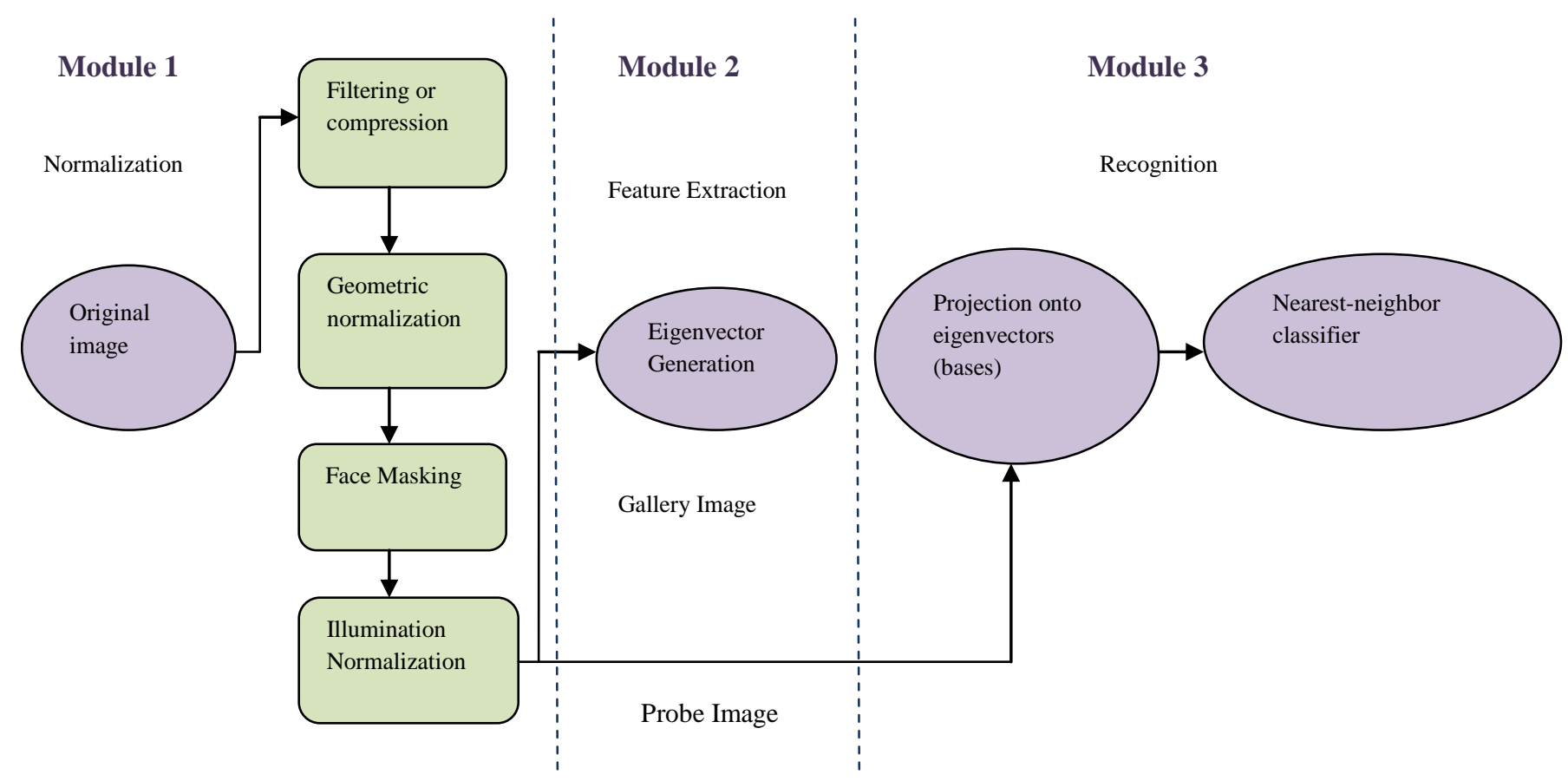

Figure 1. Block diagram of PCA Based face recognition System 


\subsection{Linear Discriminant Analysis (LDA)}

Linear Discriminant Analysis (LDA) finds the vectors in the underlying space that best discriminate among classes. For all samples of all classes the between-class scatter matrix $S B$ and the within-class scatter matrix $S \mathrm{~W}$ are defined. The goal is to maximize $S \mathrm{~B}$ while minimizing $S \mathrm{~W}$, in other words, maximize the ratio:

\section{Maximize Ratio $=\operatorname{det}|S B| / \operatorname{det}|S W|$}

This ratio is maximized when the column vectors of the projection matrix are the eigenvectors of $\left(S \mathrm{~W}^{\wedge}-1 \times S \mathrm{~B}\right)$.LDA is used for feature extraction and SVM (support vector machine) used for classification. One of the author [9] introduced the combination of PCA, LDA and NCC (Nearest Neighbor Classifier) enhancing the rate of face recognition. A comparative study of PCA and LDA for feature extraction [10]. Two parameters time and accuracy were used for evaluation in the proposed method which was conducted over six face images datasets with distinct distortion. The experimental result showed LDA is much better than PCA in terms of accuracy on the other hand in terms of time evaluation PCA is faster than LDA. A novel Hybrid Approach is used to Generalized Two-Dimensional Fisher's Linear Discriminant (HAFR G-2DFLD) Method of Face Recognition [11]. Only few regions of whole face images are changes because of distinct pose, expression, illumination, etc. To cope these facial changes, face images are segmented into sub-images and then G-2DFLD method is applied to extract local discriminant feature from sub-images and global discriminant feature from whole images. Using extracted feature a multi-class SVM classifier recognizes the subimages as well as whole image.

RECOMMENDATIONS: In features extraction, LDA generates much accurate result than PCA and it also identifies the trained persons and rejects the untrained persons in ORL database. LDA features provide better performance but in case of Indian face database its performance is reduced. If the variations of pose in database are less than it perform better verification and identification task.

\subsection{Gabor Wavelet Feature Based Approach}

In color images author [12] addressed a biometric system for face detection and recognition. The proposed algorithm automatically detect face features i.e. eyes, nose and mouth and then extract their geometrical points and for recognition, wavelet component set was used which described these fiducial points. For this, neural network is used and its performance is studied over distinct inputs. For recognition an author compared two types of features. First one is geometric distances and second one is Gabor coefficients. In proposed algorithm presents the facial features localization and extraction of their characteristic points. The facial features are categorized in three parts: Eyes localization, Nose localization and Mouth localization. Obtain 6 points characterizing two eyes by extracting four external points which represent each maximal area of stains represent each eye. Also determine the center of each eye by using center of gravity of these white stains. Second, Mouth is the bottom part of the face region and the chrominance component $\mathrm{Cr}$ depends on the red color [13] i.e. use for mouth location. To extract the extreme points of mouth, Sobel filter is use to detect contour or geometrical points of mouth. After the detection of the mouth and eyes, The nose is localize between eyes and mouth and its geometrical points are also extracted by using Sobel filter. The elements of the facial feature vector represent significant distances between all extracted points. These distances shown in fig 2. In this paper author compare two types of features i.e. geometric distances and Gabor coefficient, the result shows that Gabor cofficent are more powerful than geometric distances.

The facial feature vector is :

$V=[$ Dcenter_eye $;$ Deye $;$ Dinterior_eye $;$ Dnose $;$ Deye_nose Dmouth; Dnose_mouth]

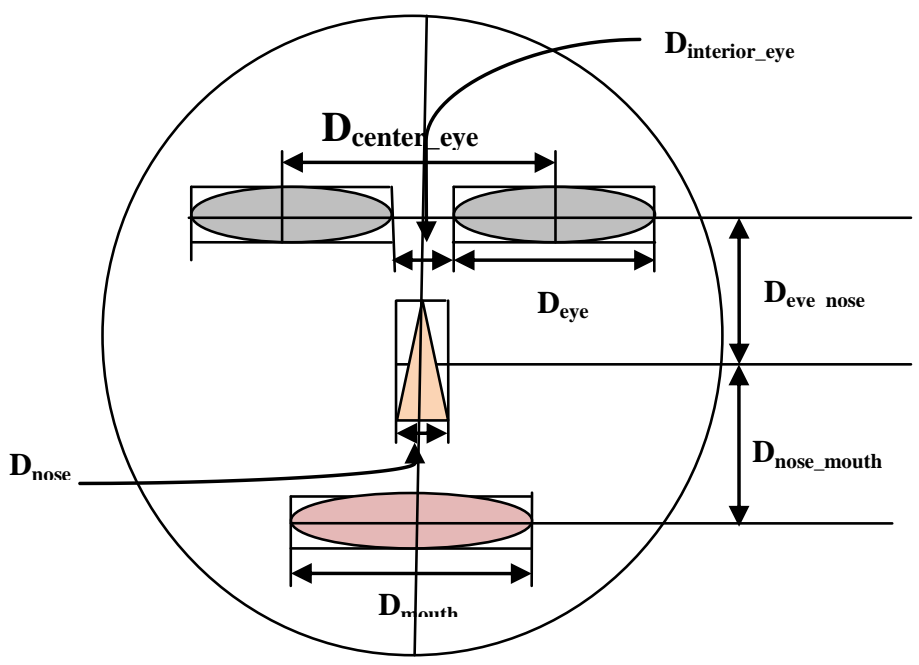

Figure 2. Combonents of facial features vector

An Author [14] introduced a new methodology in the place of raster image, to enhance face recognition rate by fusing the phase and magnitude of Gabor's representations of the face as a new representation. For this, principal component Analysis (PCA) was used as a face recognition algorithm and Eigenface was used to extract the global information. The result showed that the combination of the magnitude with the phase of Gabor features can achieve promising results. In face recognition the main issue is what features used to represent a face, the proposed algorithm extract 17 fiducial points i.e. automatically located by Active Appearance (AAMs) and characterized with Gabor wavelet analysis [14]. These features evaluated by a face database, Under some the result shows that the proposed algorithm is effective and robust.

RECOMMENDATIONS: Gabor wavelet feature based approach is better than others. In Indian face database that has more posture variation in comparison to other face database like ORL and Yale face database, the Gabor feature perform the better recognition rate from trained individuals and verification task with more variations in comparison to LDA.

\subsection{Miscellaneous Approach}

In paper [15], presents the state space feature parameters to model face images and also present feature extraction methods which recognize gray scale based face images i.e. removing any step of preprocessing. The credibility of the proposed method showed by drawing distribution graph between state space map and state space point. From state space map also computed fractal dimension by showing the nonlinear nature of face images by using box count method. K-NN classifier and SSPD are used at recognition stage for finding features. Another author [16] addressed log-polar transform and discrete cosine transform for invariant face recognition from face rotation. The discrete cosine transform used to eliminate row shifted effect and to produced low- 
dimensional feature vector and used log-polar transform to eliminate the rotation effect and then generated a row shifted log-polar image. To find the feature vector space for the optimal feature subset a particle swarm optimization based feature selection algorithm is used. Evolution is driven by a fitness function defined in terms of maximizing the betweenclass separation (scatter index). The experimental result of the proposed feature selection algorithm was found to generate excellent recognition rate i.e. $97 \%$ for the rotated test images with the minimal set of selected features. The authors [17] proposed a new feature extraction technique with respect to scale variant and rotate images. In this paper new HMAX and original HMAX model were used. The process of identification was categorized into some stages which are capturing image, preprocessing image, extract the face from image and eliminate noise and extracting feature from extracted face. For this two classifier are used i.e. K-nearest neighbor (KNN) and support vector machine (SVM). The presented technique is effective in terms of recognition rate of the system. Rahman, S. et.al [18] proposed a method for face recognition. For this face represent by using curvelet texture features, these features were computed by standard deviation of transformed and low order statistics (e.g. mean). Also support different resolution face images as input and then build the classifier and PCA is used to represent the subdivisions of features. This paper tests the system with curvelet transform and dividing the face image into different number of sub-divisions on three standard databases.

\section{Conclusion And Future Work:}

Several researches had been done for face recognition by using feature extraction. In proposed paper, have analyzed many approaches by various researchers who worked on various parameters on feature extraction. According to some author PCA provide fact computation in constraint environment; according to some author introduced LDA produced much accurate result. But in case of more variation in face posture the Gabor Wavelet feature extraction is better. Also given our recommendation to the readers as to which approach is better than others. In future we will also build an approach for face recognition using feature extraction.

\section{REFERENCES}

[1] IS THERE ANY HOPE FOR FACE RECOGNITION?,Luis Torres, Technical University of Catalonia, Barcelona, Spain.

[2] Teja, G.P.; Ravi, S., "Face recognition using subspaces techniques", Recent Trends In Information Technology (ICRTIT), 2012 International Conference.

[3] Jafri, Rabia; Arabnia, Hamid R, "Analysis of Subspacebased Face Recognition Techniques under Changes in Imaging Factors" ITNG '07. Fourth International Conference on, Publication Year: 2007 , Page(s): $406-$ 413.

[4] Liu Zunxiong; Zeng Lihui; Zhang Heng, “AppearanceBased Subspace Projection Techniques for Face Recognition" Intelligent Interaction and Affective Computing, 2009. ASIA '09. International Asia Symposium, Page(s): $202-205$.

[5] Patil, A.M.; Kolhe, S.R.; Patil, P.M. " Face Recognition by PCA Technique" Emerging Trends in Engineering and Technology (ICETET), 2nd International Conference on 2009, Page(s): $192-195$.

[6] Rajkumar, N.; Vijayakumar, S.; Murukesh, C "Intellectually combined face recognition using curvelet based principle component analysis for feature extraction and Bayesian Classifier" Signal Processing, Communication, Computing and Networking Technologies (ICSCCN), 2011 International Conference ,Page(s): $374-378$.

[7] Xiaozhe Wang; Jinping Wang; Chenyang Li" Studies on human face recognition based on greedy kernel principal component analysis "Control and Decision Conference (CCDC), 2012 24th Chinese Year: 2012 , Page(s): $1446-1449$.

[8] Ebied, H.M." Feature extraction using PCA and KernelPCA for face recognition "Informatics and_Systems (INFOS), 2012 8th International Conference_2012, Page(s): MM-72 - MM-77.

[9] Jianke Li; Baojun Zhao; Hui Zhang; Jichao Jiao" Face Recognition System Using SVM Classifier and Feature Extraction by PCA and LDA Combination" Jiao Computational Intelligence and Software Engineering, 2009. CiSE 2009. International Conference 2009 , Page(s): $1-4$.

[10] Hidayat, E.; Fajrian, N.A.; Muda, A.K.; Huoy, C.Y.; Ahmad, S. "A comparative study of feature extraction using PCA and LDA for face recognition". Information Assurance and Security (IAS), 2011 7th International Conference Publication Year: 2011 , Page(s): 354 - 359.

[11] Chowdhury, S.; Sing, J.K.; Basu, D.K.; Nasipuri, M. "A Hybrid Approach to Face Recognition Using $1 G e n e r a l i z e d$ Two-Dimensional Fisher's Linear Discriminant Method “. Emerging Trends in Engineering and Technology (ICETET), 2010 3rd International Conference Publication Year: 2010 , Page(s): 506-511.

[12] Yousra BEN JEMAA; Sana KHANFIR "Automatic local Gabor features extraction for face recognition" (IJCSIS) International Journal of Computer Science and Information Security,Vol. 3, No. 1, 2009.

[13] R. L. Hsu, M. A. Mottaleb and A. K. Jain, Face detection in color images, IEEE Trans on Pattern Analysis and Machine Intelligence, Vol.24, No.5, pp. 696-706, May 2002.

[14] Bellakhdhar, F.; Bousselmi, M.; Abid, M. "Face identification using the magnitude and the phase of Gabor wavelets and PCA". Multimedia Computing and Systems (ICMCS), 2012 International Conference, Publication Year: 2012 , Page(s): $244-249$.

[15] Kabeer, V.; Thasleema, T.M.; Narayanan, N.K.," Face Recognition Using State Space Parameters and K-NN Classifier". Innovations in Information Technology, 2007. IIT '07. 4th International Conference Publication Year: 2007 , Page(s): $476-480$.

[16] Abdel-Kader, R.F.; Ramadan, R.M.; Rizk, R.Y.,” A hybrid rotation-invariant face recognition system using Log-Polar Transform". Signal Processing and Information Technology (ISSPIT), 2009 IEEE International Symposium on, Publication Year: 2009 , Page(s): 585 - 590.

[17] Yaghoubi, Z.; Faez, K.; Eliasi, M.; Motamed, S., "Face recognition using HMAX method for feature extraction and support vector machine classifier " Image and Vision Computing New Zealand, 2009. IVCNZ '09. 24th International Conference Publication Year: 2009 , Page(s): 421 - 424

[18] Rahman, S.; Naim, S.M.; Al Farooq, A.; Islam, M.M.," Curvelet texture based face recognition using Principal Component Analysis "Computer and Information Technology (ICCIT), 2010 13th International Conference, Publication Year: 2010 , Page(s): 45 - 50 . 